\title{
Whole-body diffusion-weighted imaging with background body signal suppression in the detection of osseous and extra-osseous metastases
}

\author{
Ahmed Abdel Razek ${ }^{1 A, B, C, D, E, F, G}$, Ahmed Tawfik ${ }^{1 C, D}$, Mariam Abdel Rahmann ${ }^{1 C, E, F}$, Saleh Teima ${ }^{2 C, D}$ \\ 'Department of Diagnostic Radiology, Mansoura Faculty of Medicine, Mansoura University, Mansoura, Egypt \\ 2Department of Radiotherapy, Mansoura Faculty of Medicine, Mansoura University, Mansoura, Egypt
}

\section{Abstract}

Purpose: To assess the reproducibility of detection of osseous and extra-osseous metastases in cancer patients using whole-body diffusion-weighted imaging with background body signal suppression (WB-DWIBS).

\begin{abstract}
Material and methods: A prospective study was conducted on 39 consecutive patients ( 21 females, 18 males; mean age 48 years) with metastases, who underwent WB-DWIBS on a 1.5-T MR scanner. Image analysis was performed independently by two blinded observers. Inter-observer agreement was assessed for the detection of osseous (spinal, appendicular) and extra-osseous (hepatic, pulmonary, nodal, and peritoneal) metastases.

Results: The overall inter-observer agreement of WB-DWIBS in the detection of osseous and extra-osseous metastases was excellent $(\kappa=0.887$, agreement $=94.44 \%, p=0.001)$. There was excellent inter-observer agreement of both observers for the detection of osseous spinal $(\kappa=0.846$, agreement $=92.3 \%)$, osseous appendicular $(\kappa=0.898$, agreement $=94.8 \%)$, hepatic $(\kappa=0.847$, agreement $=92.3 \%)$, pulmonary $(\kappa=0.938$, agreement $=97.4 \%)$, nodal metastases $(\kappa=0.856$, agreement $=94.9 \%)$, and peritoneal metastasis $(\kappa=0.772$, agreement $=94.9 \%)$.
\end{abstract}

Conclusion: We concluded that WB-DWIBS is reproducible for detection of osseous and extra-osseous metastases in cancer patients.

Key words: metastases, whole-body, DWIBS, diagnosis, staging.

\section{Introduction}

Early detection of metastasis in cancer patients is an essential step for timely management and a positive outcome. Osseous metastasis can be spinal or appendicular and extra-osseous metastasis can involve the liver, lung, and lymph nodes [1-4]. Different imaging modalities are used for the detection of osseous and extra-osseous metastasis. Imaging modalities used for staging of cancer patients vary according to the local availability and expertise. Osseous metastases are screened for by whole-body imaging. They are not ruled out by various modalities because of false negatives and poor sensitivity of some studies [3-5]. Positron-emission computed tomography (PET-CT) is the method of choice because of its sensitivity; however, it is expensive and not available in most centres [5]. CT scans of the chest, abdomen, and pelvis are commonly used for assessment of extra-osseous metastasis, but they are associated with radiation exposure [6-8].

Diffusion-weighted imaging could be readily added to conventional imaging and adds valuable functional information about tumour cellularity. The rationale for use of diffusion-weighted imaging is that malignant tumours have higher cellular density than normal tissue and show restricted diffusion. Diffusion-weighted magnetic resonance (MR) imaging used in the assessment of osseous and other lesions such as liver, breast, chest tumours, and bony and soft tissue [9-12]. Recently there

Correspondence address:

Ahmed Abdel Razek, Department of Diagnostic Radiology, Mansoura Faculty of Medicine, Mansoura University, Mansoura, Egypt, 13551,

phone: 00201061948567 , e-mail: arazek@mans.edu.eg

Authors' contribution:

A Study design · B Data collection · C Statistical analysis · D Data interpretation · E Manuscript preparation · F Literature search · G Funds collection 
has been growing interest in the use of whole-body MR imaging with diffusion-weighted imaging for detection of metastases $[11,12]$. The introduction of diffusionweighted imaging with background body signal suppression (DWIBS) technique has greatly improved wholebody MR imaging [11-14]. Few studies have addressed the potential role of whole-body diffusion-weighted imaging (WB-DWI) with background body signal suppression for the detection of osseous metastases in cancer staging [15-21]. Good inter-observer agreement of this technique is a prerequisite for its widespread clinical application. A unique aspect of this study is that we have assessed the osseous and extra-osseous metastases in cancer patients in the same work.

This prospective study was performed to assess the inter-observer agreement of WB-DWIBS for the detection of osseous and extra-osseous metastases in cancer patients.

\section{Material and methods}

\section{Patients}

The institutional review board approved this study and informed consent was taken from each patient beforehand. This prospective study included 42 patients with pathologically proven primary malignant tumours and clinically suspected or known metastases. We excluded three patients from the study, due to claustrophobia in two patients and inadequate image quality in one patient due to motion artefacts. The final participants comprised 39 patients (21 females, 18 males; mean age 48 years). The primary malignant tumours were prostate cancer $(n=14)$, breast cancer $(n=12)$, colon cancer $(n=10)$, and pelvic cancer $(n=3)$. All patients underwent whole-body MR imaging using whole-body short tau inversion recovery (WB-STIR) and WB-DWIBS.

\section{Magnetic resonance imaging technique}

Whole-body MR imaging was performed using a 1.5-Tesla MR scanner (Ingenia, Philips healthcare, Best, Netherlands), using a table moving technique. Integrated body coils were used, and images were acquired in the coronal plane. Patients were examined in the supine feet-first position on a rolling table plate. Positioning of the upper extremities was according to the patient's habitus. In cachectic patients, the arms were easily placed over the thorax and abdomen. In larger patients, the arms were placed above the head, requiring an additional coronal acquisition and additional four minutes of scan time.

At first, three-plane localiser scout views were performed for the region of interest. The WB-STIR and WB-DWIBS were done in coronal plane for five separate regions: 1 - head, neck, chest apex, proximal upper limb, and cervical spine; 2 - chest, upper abdomen, upper limb, dorsal, and upper lumbar spine; 3 - lower abdomen and upper pelvis; 4 - lower pelvis and thighs; 5 - distal femur, knee joint, and proximal tibia. By the combination of the moving tabletop, table extender, and image-melding software the scan time was about 30-35 min.

The parameters of WB-STIR were: repetition time $(\mathrm{TR})=3000-5000 \mathrm{~ms}$; echo time $(\mathrm{TE})=70 \mathrm{~ms}$; inversion time $(\mathrm{TI})=165 \mathrm{~ms}$; slice thickness $=6 \mathrm{~mm}$; field of vein $($ FOV $)=300-360 \mathrm{~mm}$ and matrix $=256 \times 256$. The WB-DWIBS was done using no-EPI diffusion-weighted technique with free breathing. The parameters of WBDWIBS were: $\mathrm{TR}=7410 \mathrm{~ms}$, TE $=60 \mathrm{~ms}$, TI $=165 \mathrm{~ms}$, slice thickness $=3 \mathrm{~mm}, \mathrm{~B}$-value $=400-800 \mathrm{~s} / \mathrm{mm}^{2}$, matrix $=256 \times 256$, and $\mathrm{FOV}=250 \mathrm{~mm}$.

\section{Image analysis}

Post processing was performed offline to produce wholebody images using a Philips workstation with commercially available software. Two radiologists fully blinded to the clinical data and results of other diagnostic imaging modalities independently analysed the WB-DWIBS image sequence. Image interpretation was directed at detection of osseous and extra-osseous metastases. On WB-DWIBS images, each site of abnormal or focal increase of signal intensity not from normal anatomic structure or with an unexpected increase of signal intensity was considered as positive for metastases.

\section{Reference standard}

The standard of reference was biopsy with histopathological examination for nodal sampling $(n=12)$, hepatic focal lesions $(n=10)$, pulmonary nodules $(n=7)$ and. For verification of the other bony and extra-bony lesions detected at WB-DWIBS, a combined reference standard was used including the clinical follow-up data with a mean follow-up period of $6.2 \pm 1.2$ months for all patients, as well as progression or regression of the metastatic foci in response to the therapy at follow-up CT.

\section{Statistical analysis}

The statistical analysis of data was done using the Statistical Package for Social Science, version 22 (SPSS Inc., Chicago, IL, USA). The inter-observer agreement was assessed by kappa ( $\kappa)$ statistic with $95 \%$ confidence intervals (CI); a $p$ value $<0.05$ was considered to indicate statistical significance. The $\kappa$ coefficient is the amount of observed agreement. A $\kappa$ of 1.0 represents perfect agreement, a $\kappa$ of 0.81 to 1.0 is excellent agreement, and a $\kappa$ of 0.61 to 0.80 is good agreement.

\section{Results}

Table 1 shows inter-observer agreement of WB-DWIBS in the detection of osseous and extra-osseous metastatic 
Table 1. Inter-observer agreement of whole-body diffusion-weighted imaging with background body signal suppression (WB-DWIBS) in detection of osseous and extra-osseous metastases

\begin{tabular}{|l|c|c|c|c|c|c|}
\hline Metastases & Observer 1 & Observer 2 & Percentage agreement & $\kappa$ & $95 \% \mathrm{Cl}$ & $p$ value \\
\hline Osseous & 38 & 37 & $93.6 \%$ & 0.872 & $0.76-0.98$ & 0.001 \\
\hline Spinal & 18 & 19 & $92.3 \%$ & 0.846 & $0.68-1.0$ & 0.001 \\
\hline Appendicular & 20 & 18 & $94.8 \%$ & 0.898 & $0.76-1.0$ & 0.001 \\
\hline Extra-0sseous & 67 & 65 & $94.9 \%$ & 0.895 & $0.82-0.97$ & 0.001 \\
\hline Hepatic & 18 & 21 & $92.3 \%$ & 0.847 & $0.68-1.0$ & 0.001 \\
\hline Pulmonary & 12 & 11 & $97.4 \%$ & 0.938 & $0.82-1.0$ & 0.001 \\
\hline Nodal & 31 & 29 & $94.9 \%$ & 0.856 & $0.66-1.0$ & 0.001 \\
\hline Peritoneal & 6 & 4 & $94.9 \%$ & 0.772 & $0.50-1.0$ & 0.001 \\
\hline Overall & 105 & 102 & $94.44 \%$ & 0.887 & $0.83-0.94$ & 0.001 \\
\hline
\end{tabular}
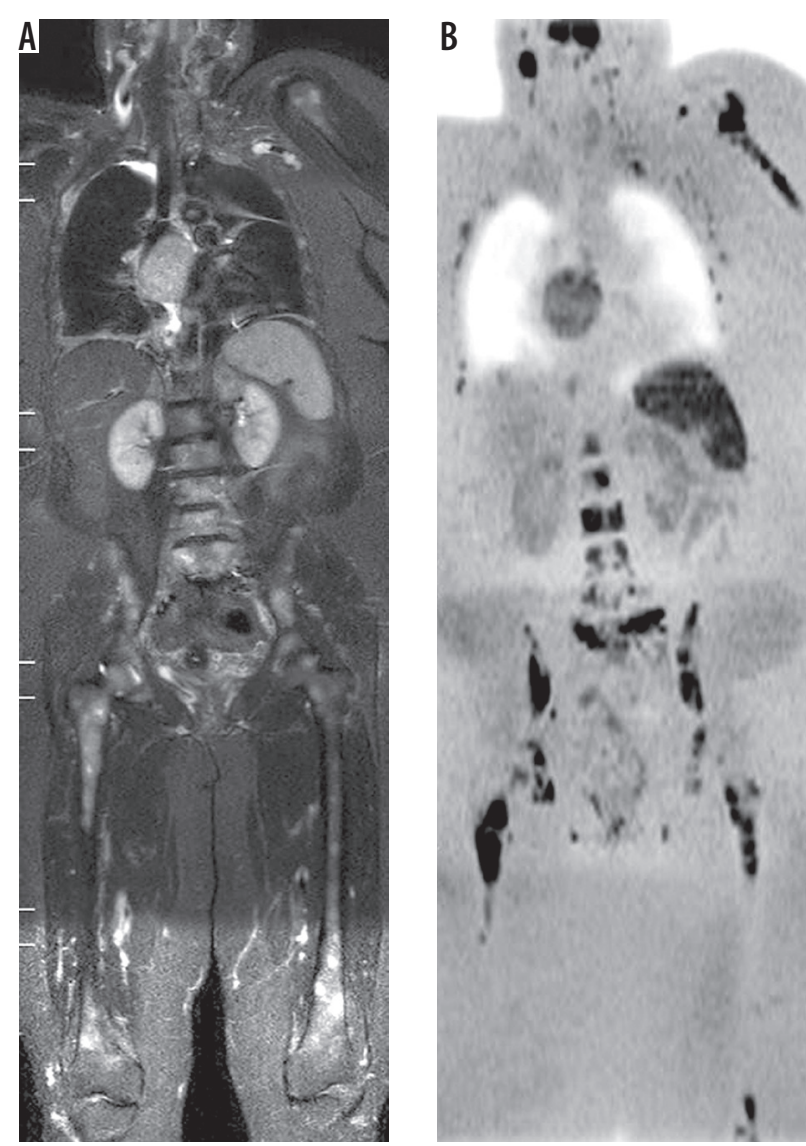

Figure 1. Spinal and appendicular metastases from breast cancer: A) Wholebody short tau inversion recovery image shows multiple well-defined hyper-intense lesions seen in both femori and the spine. B) Whole-body diffusion-weighted imaging with background body signal suppression image shows better evaluation of osseous metastasis in the spine, both femurs, left scapula, and ribs with restricted diffusion of the lesion

deposits in cancer patients. The overall inter-observer agreement of WB-DWIBS in the detection of metastases was excellent $(\kappa=0.887$, agreement $=94.44 \%)$.

The overall inter-observer agreement of WB-DWIBS in the detection of osseous metastases was excellent $(\kappa=0.872$, agreement $=93.6 \%)$. Osseous spinal metastases (Figure 1) were reported in 18 patients by observer 1 and 19 patients
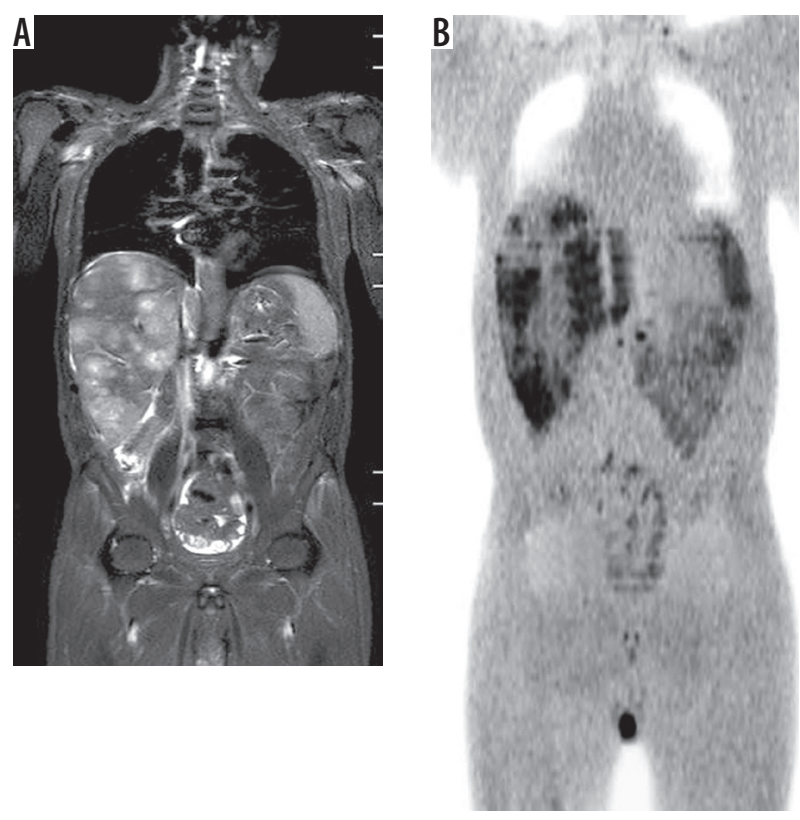

Figure 2. Hepatic metastases from colon cancer. A) Whole-body short tau inversion recovery image shows multiple hyper-intense focal lesions of the liver. B) Whole-body diffusion-weighted imaging with background body signal suppression image shows multiple focal lesions of the liver with restricted diffusion

by observer 2 , with an excellent agreement $(\kappa=0.846$, agreement $=92.3 \%)$. Osseous appendicular metastases (Figure 1) were reported in 20 patients by observer 1 and 18 patients by observer 2 , with an excellent agreement $(\kappa=0.898$, agreement $=94.8 \%)$.

The overall inter-observer agreement of WB-DWIBS in the detection of extra-osseous metastases was excellent $(\kappa=0.895$, agreement $=94.9 \%)$. Hepatic metastases (Figure 2) were reported in 18 patients by observer 1 and 21 patients by observer 2 , with excellent agreement $(\kappa=0.847$, agreement $=92.3 \%$ ). Pulmonary metastases were reported in 12 patients by observer 1 and 11 patients by observer 2 , with excellent agreement $(\kappa=0.938$, agreement $=97.4 \%)$. Nodal metastases (Figure 3 ) were reported in 31 patients by observer 1 and in 29 patients by observer 2, with excel- 

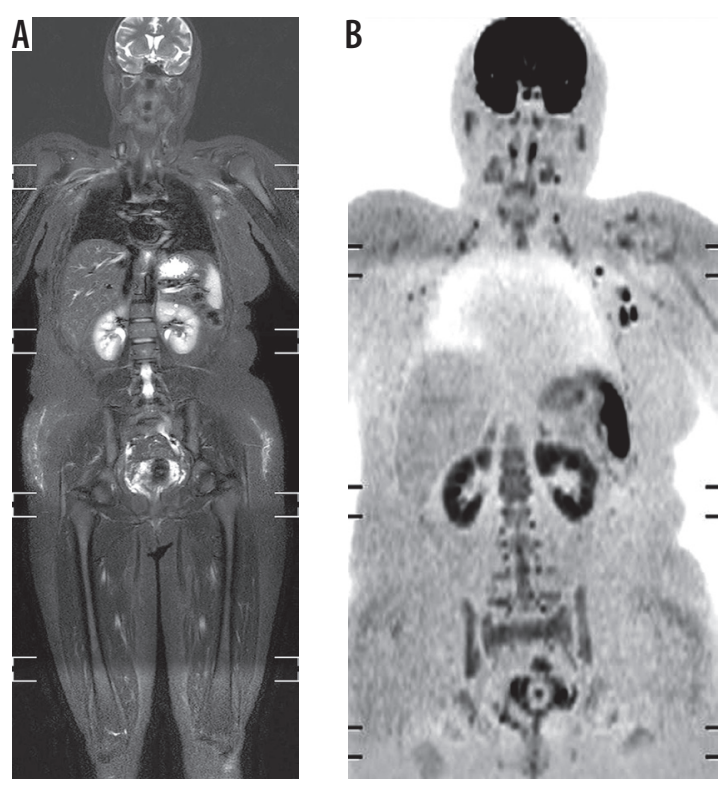

lent agreement $(\kappa=0.856$, agreement $=94.9 \%)$. Peritoneal metastases were reported in six patients by observer 1 and in four patients by observer 2, with excellent agreement $(k=0.772$, agreement $=94.9 \%)$.

\section{Discussion}

The main finding in this study is that there is excellent inter-observer agreement of WB-DWIBS $(\kappa=0.887)$ in the overall detection of metastases, including osseous spinal or appendicular metastases as well as extra-osseous metastases, whether hepatic, pulmonary, nodal, or peritoneal.

There are several studies that have compared WB-MRI to skeletal scintigraphy for the detection of osseous metastases, with encouraging results about the performance of WB-MRI. In a study by Stecco et al., the accuracy of WB-DWIBS was similar to scintigraphy, with excellent agreement between the two methods [22]. Gutzeit et al. reported that WB-DWIBS detected more metastases than scintigraphy in patients with large numbers of lesions [23]. Lecouvet et al. reported that the performance of WB-DWIBS is better than scintigraphy in the detection of osseous metastases in patients with high-risk prostate cancer [24]. Similarly, the diagnostic accuracy of WB-MRI for detection of bone metastases was significantly higher than skeletal scintigraphy [25].

In this study, the inter-observer agreement of WBDWIBS in the detection of spinal and appendicular osseous metastases is excellent ( $\kappa=0.846$ and 0.898 , respectively). Balliu et al. reported excellent agreement $(\kappa=0.895)$ for WB-MRI in metastases detection [25]. On the other hand, earlier studies reported that the inter-observer agreement of WB-DWIBS is good $(\kappa=0.66,0.68)$ [22-33].

In this study, there was excellent inter-observer agreement in the detection of hepatic $(\kappa=0.847)$ and pulmonary $(\kappa=0.938)$ metastases, and good agreement for peritoneal $(\kappa=0.772)$ metastases. Stecco et al. reported

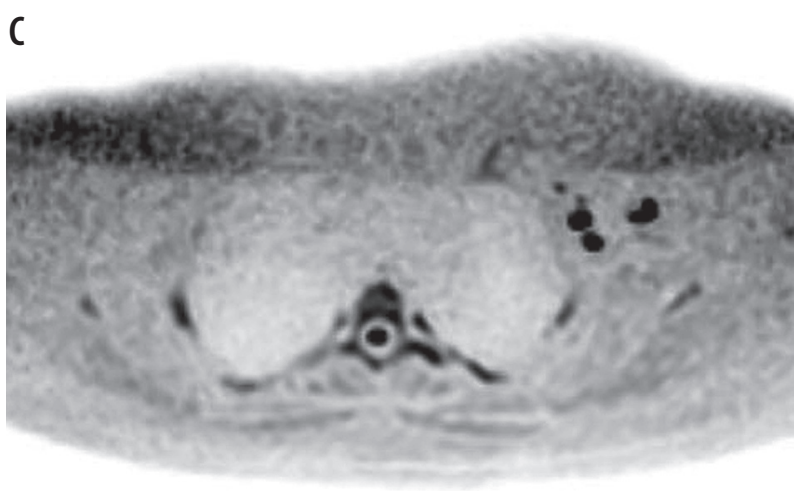

Figure 3. Nodal metastases from breast cancer after mastectomy. A) Wholebody short tau inversion recovery image shows well-defined hyper-intense left-sided axillary lymph nodes. B, C) Whole-body diffusion-weighted imaging with background body signal suppression shows restricted diffusion of the left axillary lymph nodes

excellent agreement $(\kappa=0.93)$ between two observers using WB-DWIBS in the staging of oncologic patients [15]. One study reported that the accuracy of WB-DWI for the detection of pulmonary metastases is only $60 \%$ compared to $100 \%$ for conventional CT [26]. Another study reported that the sensitivity of WB-DWIBS for the detection of pulmonary metastases is lower than that of CT (51.6\% vs. $71.4 \%$, respectively) [27].

In this study, the agreement between both observers for nodal metastasis was excellent $(\kappa=0.856)$. Visual assessment by WB-DWIBS is well suited for the detection of lymph nodes because of the cellularity of nodes in contrast to the suppressed signal of the surrounding fat [14]. One study reported that WB-DWIBS has the same diagnostic performance as combined CT, PET-CT, and lymph node ultrasonography in the detection of nodal metastases [27]. Diffusion characteristics should be supplemented by morphological data of the size and shape criteria of the lymph node. Few studies discuss the value of apparent diffusion coefficient (ADC) measurements for the identification of nodal metastases. These studies reported that malignant lymph nodes reported restricted diffusion with lower ADC values the than benign nodes [28-34].

The implementation of WB-DWIBS in whole-body imaging has many advantages. This technique allowed shorter interpretation time and easy visual assessment with reconstructed multi-planar images. This technique uses free breathing and therefore enables thin axial section acquisition and multiple signal averaging, in addition to fat-suppression, which further highlights regions of diffusion restriction [14-17]. Previous studies demonstrated the high accuracy of WB-DWIBS in the staging of cancer patients in comparison to positron-emission computed tomography $[15,17,19]$. WB-DWIBS has a great potential for whole-body imaging of oncologic patients, being faster and more convenient for patients than undergoing multiple imaging sessions for staging, with substantial cost 
and time savings [2-4]. The lack of radiation and injection of contrast medium or isotope make WB-DWIBS well suited for screening [11-14].

The ability of WB-DWIBS for metastases detection is variable and is affected by many factors including the histological type and grade of the tumour as well as the anatomic location of the target lesions. False negative results may also be seen with tumours located in sites affected by motion that causes decreased signal, such as the lung hila, mediastinum, or the uppermost part of the left hepatic lobe [12-15]. Susceptibility artefacts represent another challenge that may degrade image quality and mask lesions, leading to false negative results [11]. False positive results may be obtained due to the so-called "T2-shine through" effect, where lesions with high T2 signal may be mistaken for areas of restricted diffusion [1-4].

There are a few limitations of this study. First, the number of patients is relatively small. Further studies on a larger cohort are encouraged. Second, we admit patients have been not examined by PET/CT. Further studies are recommended to correlate osseous and extra-osseous lesions detected at WB-DWIBS with PET-CT findings. Third, this study applies diffusion-weighted imaging on 1.5-Tesla scanners. Further studies applied to diffusion tensor imaging combined with other sequences of MR such as arterial spin labelling on higher 3-Tesla scanners will improve the results in the future [35-46].

\section{Conclusions}

We conclude that the WB-DWIBS technique is reproducible for the detection of osseous and extra-osseous metastases in cancer patients.

\section{Disclosure}

The authors declare no conflicts of interest.

\section{References}

1. Bezerra ROF, Recchimuzzi DZ, Dos Santos Mota MM, et al. Wholebody magnetic resonance imaging in the oncology setting: an overview and update on recent advances. J Comput Assist Tomogr 2019; 43: 66-75.

2. Barnes A, Alonzi R, Blackledge M, et al. UK quantitative WB-DWI technical workgroup: consensus meeting recommendations on optimisation, quality control, processing and analysis of quantitative whole-body diffusion-weighted imaging for cancer. Br J Radiol 2018; 91: 20170577.

3. Morone M, Bali MA, Tunariu N, et al. Whole-body MRI: current applications in oncology. AJR Am J Roentgenol 2017; 209: W336349.

4. Han SN, Amant F, Michielsen K, et al. Feasibility of whole-body diffusion-weighted MRI for detection of primary tumour, nodal and distant metastases in women with cancer during pregnancy: a pilot study. Eur Radiol 2018; 28: 1862-1874.

5. Petralia G, Padhani AR, Pricolo P, et al. Whole-body magnetic resonance imaging (WB-MRI) in oncology: recommendations and key uses. Radiol Med 2019; 124: 218-233.

6. Razek AA, Ezzat A, Azmy E, et al. Role of whole-body 64-slice multidetector computed tomography in treatment planning for multiple myeloma. Radiol Med 2013; 118: 799-805.

7. Abdel Razek A, Shamaa S, Abdel Lattif M, et al. Inter-observer agreement of whole-body computed tomography in staging and response assessment of lymphoma: the Lugano classification. Polish J Radiol 2017; 82: 441-447.

8. Ghobrial FE, Salah Eldin M, Razek AA, et al. Computed tomography assessment of hepatic metastases of breast cancer with revised response evaluation criteria in solid tumors (RECIST) criteria (Version 1.1): inter-observer agreement. Polish J Radiol 2017; 82: 593-597.

9. Razek AA. Diffusion magnetic resonance imaging of chest tumors. Cancer Imaging 2012; 12: 452-463.
10. Razek AA, Gaballa G, Denewer A, Tawakol I. Diffusion weighted MR imaging of the breast. Acad Radiol 2010; 17: 382-386.

11. Lecouvet FE. Whole-body MR imaging: musculoskeletal applications. Radiology 2016; 279: 345-365.

12. Koh DM, Blackledge M, Padhani AR, et al. Whole-body diffusionweighted MRI: tips, tricks, and pitfalls. AJR Am J Roentgenol 2012; 199: $252-262$

13. Padhani AR, Koh DM, Collins DJ. Whole-body diffusion-weighted MR imaging in cancer: current status and research directions. Radiology 2011; 261: 700-718.

14. Kwee TC, Takahara T, Ochiai R, et al. Diffusion-weighted wholebody imaging with background body signal suppression (DWIBS): features and potential applications in oncology. Eur Radiol 2008; 18: 1937-1952.

15. Stecco A, Romano G, Negru M, et al. Whole-body diffusion-weighted magnetic resonance imaging in the staging of oncological patients: comparison with positron emission tomography computed tomography (PET-CT) in a pilot study. Radiol Med 2009; 114: 1-17.

16. Kalkmann J, Lauenstein T, Stattaus J. Whole-body diffusion-weighted imaging in oncology. Technical aspects and practical relevance. Radiologe 2011; 51: 215-219.

17. Manenti G, Cicciò C, Squillaci E, et al. Role of combined DWIBS/ 3D-CE-T1w whole-body MRI in tumor staging: comparison with PET-CT. Eur J Radiol 2012; 81: 1917-1925.

18. Wilhelm T, Stieltjes B, Schlemmer HP. Whole-body-MR-diffusion weighted imaging in oncology. Rofo 2013; 185: 950-958.

19. Li B, Li Q, Nie W, et al. Diagnostic value of whole-body diffusion-weighted magnetic resonance imaging for detection of primary and metastatic malignancies: a meta-analysis. Eur J Radiol 2014; 83: 338-344.

20. Huppertz A, Schmidt M, Wagner M, et al. Whole-body MR imaging versus sequential multimodal diagnostic algorithm for staging patients with rectal cancer: cost analysis. Rofo 2010; 182: 793-802. 
21. Tarnoki DL, Tarnoki AD, Richter A, et al. Clinical value of wholebody magnetic resonance imaging in health screening of general adult population. Radiol Oncol 2015; 49: 10-16.

22. Stecco A, Lombardi M, Leva L, et al. Diagnostic accuracy and agreement between whole-body diffusion MRI and bone scintigraphy in detecting bone metastases. Radiol Med 2013; 118: 465-475.

23. Gutzeit A, Doert A, Froehlich JM, et al. Comparison of diffusionweighted whole-body MRI and skeletal scintigraphy for the detection of bone metastases in patients with prostate or breast carcinoma. Skeletal Radiol 2010; 39: 333-343.

24. Lecouvet FE, El Mouedden J, Collette L, et al. Can whole-body magnetic resonance imaging with diffusion-weighted imaging replace Tc $99 \mathrm{~m}$ bone scanning and computed tomography for single-step detection of metastases in patients with high-risk prostate cancer? Eur Urol 2012; 62: 68-75.

25. Balliu E, Boada M, Peláez I, et al. Comparative study of whole-body MRI and bone scintigraphy for the detection of bone metastases. Clin Radiol 2010; 65: 989-996.

26. Paruthikunnan SM, Kadavigere R, Karegowda LH. Accuracy of whole-body DWI for metastases screening in a diverse group of malignancies: comparison with conventional cross-sectional imaging and nuclear scintigraphy. AJR Am J Roentgenol 2017; 209: 477-490.

27. Jouvet JC, Thomas L, Thomson V, et al. Whole-body MRI with diffusion-weighted sequences compared with 18 FDG PET-CT, CT and superficial lymph node ultrasonography in the staging of advanced cutaneous melanoma: a prospective study. J Eur Acad Dermatol Venereol 2014; 28: 176-185.

28. Abdel Razek AA, Soliman NY, Elkhamary S, et al. Role of diffusion-weighted MR imaging in cervical lymphadenopathy. Eur Radiol 2006; 16: 1468-1477.

29. Vandecaveye V, De Keyzer F, Vander Poorten V, et al. Head and neck squamous cell carcinoma: value of diffusion-weighted MR imaging for nodal staging. Radiology 2009; 251: 134-146.

30. Abdel Razek AA, Elkammary S, Elmorsy AS, et al. Characterization of mediastinal lymphadenopathy with diffusion-weighted imaging. Magn Reson Imaging 2011; 29: 167-172.

31. Chung J, Youk JH, Kim JA, et al. Role of diffusion-weighted MRI: predicting axillary lymph node metastases in breast cancer. Acta Radiol 2014; 55: 909-916.

32. Seber T, Caglar E, Uylar T, et al. Diagnostic value of diffusion-weighted magnetic resonance imaging: differentiation of benign and malignant lymph nodes in different regions of the body. Clin Imaging 2015; 39 : 856-862.

33. Razek AA, Lattif MA, Denewer A, et al. Assessment of axillary lymph nodes in patients with breast cancer with diffusion-weighted
MR imaging in combination with routine and dynamic contrast MR imaging. Breast Cancer 2016; 23: 525-532.

34. Abdel Razek AA, Soliman N, Elashery R. Apparent diffusion coefficient values of mediastinal masses in children. Eur J Radiol 2012; 81: 1311-1314.

35. Razek AAKA, Ashmalla GA. Assessment of paraspinal neurogenic tumors with diffusion-weighted MR imaging. Eur Spine J 2018; 27: 841-846.

36. Abdel Razek A, Mossad A, Ghonim M. Role of diffusion-weighted MR imaging in assessing malignant versus benign skull-base lesions. Radiol Med 2011; 116: 125-132.

37. Abdel Razek AAK. Routine and Advanced Diffusion Imaging Modules of the Salivary Glands. Neuroimaging Clin N Am 2018; 28: 245-254.

38. Razek AA, Nada N. Correlation of Choline/Creatine and Apparent Diffusion Coefficient values with the prognostic parameters of Head and Neck Squamous Cell Carcinoma. NMR Biomed 2016; 29: 483-489.

39. Razek AAKA. Diffusion tensor imaging in differentiation of residual head and neck squamous cell carcinoma from post-radiation changes. Magn Reson Imaging 2018; 54: 84-89.

40. El-Serougy L, Abdel Razek AA, Ezzat A, et al. Assessment of diffusion tensor imaging metrics in differentiating low-grade from highgrade gliomas. Neuroradiol J 2016; 29: 400-407.

41. Khalek Abdel Razek AA. Characterization of salivary gland tumours with diffusion tensor imaging. Dentomaxillofac Radiol 2018; 47: 20170343

42. Abdel Razek A, Zaki M, Bayoumi D, et al. Diffusion tensor imaging parameters in differentiation recurrent breast cancer from post-operative changes in patients with breast-conserving surgery. Eur J Radiol 2019; 111: 76-80.

43. Abdel Razek AAK, Talaat M, El-Serougy L, et al. Clinical applications of arterial spin labeling in brain tumors. J Comput Assist Tomogr 2019; 43: 525-532.

44. Abdel Razek AAK. Arterial spin labelling and diffusion-weighted magnetic resonance imaging in differentiation of recurrent head and neck cancer from post-radiation changes. J Laryngol Otol 2018; 132: 923-928.

45. Razek AAKA, El-Serougy L, Abdelsalam M, et al. Differentiation of residual/recurrent gliomas from postradiation necrosis with arterial spin labeling and diffusion tensor magnetic resonance imaging-derived metrics. Neuroradiology 2018; 60: 169-177.

46. Surov A, Nagata S, Razek AA, et al. Comparison of ADC values in different malignancies of the skeletal musculature: a multicentric analysis. Skeletal Radiol 2015; 44: 995-1000. 